\section{Asociación entre edulcorantes no nutritivos y riesgo de obesidad en estudiantes universitarios de Latinoamérica}

\author{
SAMUEL DURÁN AGÚERO ${ }^{1, \mathrm{a}}$, ESTELA BLANCO BATTEN ${ }^{2, \mathrm{~b}}$, \\ MARÍA DEL PILAR RODRÍGUEZ NOEL ${ }^{3, \mathrm{c}}$, \\ KARLA CORDÓN ARRIVILLAGA ${ }^{4, c}$, JULIETA SALAZAR DE ARIZA ${ }^{4, c}$, \\ JINIVA RECORD CORNWALL ${ }^{5, c}$, MARÍA DEL PILAR CERECEDA \\ BUJAICO $^{6, \mathrm{c}}$, SONIA ANTEZANA ALMORZA ${ }^{6}$, \\ SISSY ESPINOZA BERNARDO ${ }^{6}$, CLAUDIA ENCINA VEGA $^{3, c}$
}

\section{Association between non-nutritive sweeteners and obesity risk among university students in Latin America}

Background: The association between non-nutritive sweeteners and obesity is controversial. Aim: To determine whether the consumption of non-nutritive sweeteners is related to higher risk for overweight or obesity among university students in Chile, Panama, Guatemala and Peru. Material and Methods: A total of 1,224 (472 from Chile, 300 from Panama, 248 from Guatemala and 204 from Peru) male and female university students aged between 18 and 26 years participated in the study. Each student reported their food intake (frequency of weekly consumption) in a survey that contained photos of foods containing non-nutritive sweeteners adapted for each country. Anthropometry was also measured. Results: More than $80 \%$ of students consumed at least one product containing non-nutritive sweeteners. Females who ate acesulfame potassium and sucralose had a lower risk of overweight or obesity with an odds ratio (OR) of 0.5 (confidence intervals $(C I)=0.3-0.9 ; p=0.003)$ and $O R=0.4(I C=0.2-0.8$; $p=0.01)$, respectively. Conclusions: In this sample of Latinamerican university students, consumption of non-nutritive sweeteners was associated with lower risk of overweight only in females.

(Rev Med Chile 2015; 143: 367-373)

Key words: Acetosulfame; Non-nutritive sweeteners; Nutritional status; Obesity; Sucrose; Tricholorosucrose.

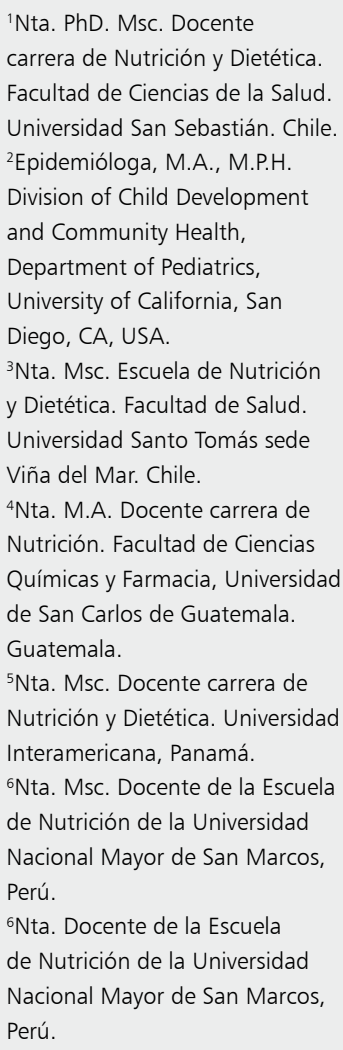

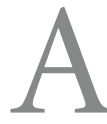
nivel mundial entre las regiones con mayor prevalencia de obesidad se encuentra Sudamérica y América Central, países en transición nutricional, donde se ha incrementado la prevalencia de hiperlipemias, sobrepeso y obesidad $^{1}$. El Îndice de Masa Corporal (IMC) en las mujeres en estas regiones aumentó 1,3 a 1,4 puntos por década, lo cual sólo fue superado por
Oceanía $^{2}$. La prevalencia de obesidad en Latinoamérica presenta una gran variabilidad que va de 9,9 a $35,7 \% \%^{3}$.

En los últimos años, el aumento en la mortalidad relacionada con la obesidad se ha traducido en un aumento de dietas para pérdida de peso, rutinas de acondicionamiento físico y diversos productos dietéticos. Entre los factores alimen- 
tarios que contribuyen a la obesidad se encuentra el elevado consumo de azúcares y grasas ${ }^{4}$. Como resultado del impacto negativo en el peso corporal con el consumo de azúcar, se ha incrementado el consumo de edulcorantes no nutritivos $(\mathrm{ENN})^{5}$, en Estados Unidos de Norteamérica ${ }^{6}$ L Latinoamérica, como una estrategia para reducir el consumo calorías $^{7-8}$.

Actualmente existe una gran variedad de ENN en el mercado, y estos son entre 30 a 13.000 veces más dulce que el azúcar9. Entre los ENN consumidos de forma habitual podemos nombrar la sacarina, aspartame, sucralosa, estevia, ciclamato y acesulfame de potasio, entre otros ${ }^{9}$. El principio fundamental detrás del consumo de los ENN es que los individuos pueden consumir alimentos o bebidas sin el riesgo de calorías adicionales aportadas por productos que contienen azúcar. Actualmente se pueden encontrar en casi cualquier alimento, tales como bebidas, helados, mermeladas, leches saborizadas y yogurt.

Sustituir el azúcar por ENN puede ser una estrategia eficaz para el control de peso corporal, aunque los resultados son contradictorios ${ }^{10-12}$. Recientemente se ha iniciado el debate acerca de si el consumo de ENN aumenta el apetito y el peso corporal ${ }^{13}$ generando confusión entre los consumidores de estos productos.

Aunque hay estudios epidemiológicos en países desarrollados que han mostrado esa asociación ${ }^{9-10}$, no hay datos a nivel de países en transición epidemiológica y nutricional que muestren la asociación entre consumo de ENN e incremento de peso.

El objetivo del presente estudio es determinar si la ingesta de ENN en universitarios de Chile, Panamá, Guatemala y Perú se asocia con un incremento en el riesgo de sobrepeso/obesidad.

\section{Material y Métodos}

La muestra estudiada corresponde a 1.224 estudiantes universitarios de ambos sexos de 18 a 26 años, de los cuales 472 fueron de Chile, 300 de Panamá, 248 de Guatemala y 204 de Perú. El tamaño de la muestra se calculó a partir del estudio de Arcella y cols ${ }^{14}$ con un intervalo de confianza de $95 \%$, una potencia de $90 \%$ y una precisión calculada como (media observada de consumo habitual de aspartame-ingesta diaria admitida para aspartame).

\section{Procedimientos}

\section{Encuestas de consumo de alimentos}

Se realizó un muestreo de los edulcorantes y alimentos que contienen ENN en el mercado de cada país, a través de visitas a mercados y supermercados, con un total de 122 productos para Chile, 109 para Panamá, 29 para Guatemala y 124 para Perú. Cada producto fue fotografiado y numerado. Sé utilizó una encuesta de frecuencia de consumo semanal de alimentos adaptada (sólo aparecen alimentos que contienen edulcorantes) y edulcorantes líquidos, en polvo o en tabletas, y validada por grupos de expertos para evaluar la ingesta de edulcorantes ${ }^{7}$. Las porciones fueron descritas como utensilios típicos de uso en el hogar (gotas, vaso, taza, cuchara, cucharadita, plato, etc.). Esta información fue utilizada para calcular la ingesta diaria de cada ENN. Las encuestas fueron aplicadas por estudiantes de Nutrición y Dietética previamente capacitados. Para evaluar la Ingesta diaria admisible (IDA), utilizamos las recomendaciones del Reglamento Sanitario de los Alimentos ${ }^{15}$.

En el presente análisis se eliminó la información sobre consumo de estevia ya que sólo estaba presente en Perú y Chile, y de ciclamato y sacarina donde el consumo estaba presente sólo en Chile.

\section{Antropometría}

El peso se midió utilizando una balanza electrónica de precisión $\left(\right.$ GAMMA $\left.^{\circledR}\right)$ con una sensibilidad de $0,1 \mathrm{~kg}$, que permitió calcular la ingesta de los edulcorantes por kilo de peso. La estatura se midió con un tallímetro que está incorporado a la balanza. El estado nutricional fue determinado con el IMC. Este índice se calculó dividiendo el peso por la talla al cuadrado (IMC = peso $\mathrm{kg} /$ talla ${ }^{2}$, y el estado nutricional se clasificó como: IMC normal $=18,5-24,9 \mathrm{~kg} / \mathrm{m}^{2}$, sobrepeso $=25,0$ a $29,9 \mathrm{~kg} / \mathrm{m}^{2}$ y obesidad mayor o igual a $30 \mathrm{~kg} /$ $\mathrm{m}^{2}$. Los antropometristas fueron estandarizados para disminuir el error intra-evaluador e interevaluador.

\section{Estadística}

Los datos fueron procesados en una planilla Excel y en el programa estadístico SPSS 19.0. Para evaluar la normalidad de las variables continuas (edad, peso, talla e ingesta de ENN) se utilizó la prueba de bondad de Kolmogorov-Smirnov. Para 
la comparación entre países se utilizó la prueba estadística ANOVA y para las variables de distribución no-normal la prueba de Kruskal-Wallis. Además, se llevó a cabo un análisis de regresión logística para evaluar la asociación existente entre el consumo de edulcorantes y el riego de obesidad, ajustando las variables por edad. En todos los casos se consideró significativo un valor de $\mathrm{p}<0,05$.

\section{Resultados}

La muestra estuvo conformada por 1.224 estudiantes de ambos sexos, de distintas carreras, como derecho, ingenierías, educación y salud. Más de $80 \%$ de los estudiantes consume al menos 1 producto que contiene un ENN de forma diaria. En la Tabla 1 se muestran las características antropométricas en los distintos países, del total de estudiantes 5,3\% de los estudiantes presentó obesidad y $21,7 \%$ sobrepeso, siendo mayor el sobrepeso y la obesidad en hombres panameños (41,6\% y $16,6 \%$ respectivamente). En la Tabla 2 se muestra los valores de ingesta diaria de los edulcorantes según país y sexo. En todos los países el edulcorante con mayor consumo entre hombres y mujeres fue aspartamo (Tabla 2). Las mujeres panameñas son las que consumen significativamente más ENN (acesulfamo de potasio y aspartamo), en cambio en los hombres las ingestas más elevadas se observan en guatemaltecos. Con respecto al Ingesta diaria admisible (IDA), ningún estudiante superó este valor.

De acuerdo a los resultados obtenidos, al realizar el análisis por todos países no se observa asociación entre el consumo de ENN y riesgo de sobrepeso/obesidad, sin embargo, las mujeres que consumen acesulfame de potasio y sucralosa presentan un menor riesgo de sobrepeso/obesidad $\mathrm{OR}=0,5(\mathrm{IC}=0,30-0,94 ; \mathrm{p}=0,031)$ y $\mathrm{OR}=0,4$ (IC $=0,24-0,80 ; p=0,008)$ que las que no consumen estos ENN. En varones no hubo asociación entre consumo de ENN y riesgo de sobrepeso/ obesidad. Al repetir los análisis para cada país, observamos que en Guatemala el consumo de acesulfame de potasio se asoció con un incremen-

Tabla 1. Comparación antropométrica en universitarios de cuatro países latinoamericanos

\begin{tabular}{|c|c|c|c|c|c|}
\hline & Total & Perú & Chile & Guatemala & Panamá \\
\hline Mujer & 885 & 140 & 317 & 212 & 216 \\
\hline Edad (años) & $22,3 \pm 4,8$ & $22,0 \pm 2,2$ & $20,9 \pm 2,5$ & $22,3 \pm 3,2$ & $24,8 \pm 7,9$ \\
\hline Peso (kg) & $58,8 \pm 9,5$ & $57,1 \pm 9,0^{a}$ & $58,8 \pm 7,7^{b}$ & $57,3 \pm 9,5^{c}$ & $61,5 \pm 11,6^{a, b, c}$ \\
\hline Talla (m) & $1,60 \pm 0,03$ & $1,57 \pm 0,06^{a, b, c}$ & $1,61 \pm 0,05^{a}$ & $1,59 \pm 0,06^{b}$ & $1,60 \pm 0,06^{c}$ \\
\hline IMC (kg/m²) & $22,8 \pm 3,4$ & $22,6 \pm 2,5^{\mathrm{a}}$ & $22,8 \pm 3,2$ & $22,3 \pm 3,6$ & $23,7 \pm 4,2^{a, b}$ \\
\hline \% Bajo peso & 5,8 & 5,0 & 1,8 & 11,5 & 6,4 \\
\hline$\%$ Normal & 71,9 & 75,0 & 78,5 & 67,7 & 64,3 \\
\hline \% Sobrepeso & 17,8 & 15,0 & 19,2 & 18,9 & 18,9 \\
\hline $\begin{array}{l}\% \text { Obesidad } \\
* * p=0,001\end{array}$ & 4,3 & 5,0 & 0,3 & 10,1 & 10,1 \\
\hline Hombre & 339 & 64 & 155 & 36 & 84 \\
\hline Edad (años) & $22,3 \pm 4,1$ & $22,6 \pm 2,9$ & $21,2 \pm 2,6$ & $23,3 \pm 3,2$ & $23,6 \pm 6,5$ \\
\hline Peso (kg) & $73,9 \pm 13,2$ & $69,1 \pm 12,3^{a}$ & $73,5 \pm 11,1$ & $74,2 \pm 12,6$ & $77,1 \pm 16,4^{a}$ \\
\hline Talla (m) & $1,72 \pm 0,07$ & $1,70 \pm 0,08^{a}$ & $1,73 \pm 0,07$ & $1,74 \pm 0,07^{a}$ & $1,72 \pm 0,08$ \\
\hline IMC (kg/m²) & $24,6 \pm 3,6$ & $23,8 \pm 2,8^{a}$ & $24,4 \pm 2,9$ & $24,2 \pm 3,2$ & $25,7 \pm 4,9^{a}$ \\
\hline \% Bajo peso & 1,4 & 1,5 & 0,0 & 2,7 & 3,5 \\
\hline \% Normal & 58,9 & 75,0 & 61,9 & 66,6 & 38,0 \\
\hline \% Sobrepeso & 31,5 & 18,7 & 32,9 & 25,0 & 41,6 \\
\hline $\begin{array}{l}\% \text { Obesidad } \\
* * p=0,001\end{array}$ & 7,9 & 4,6 & 5,1 & 5,5 & 16,6 \\
\hline
\end{tabular}

Prueba de Anovapost hoc Bonferroni, letras iguales indican diferencias significativas $(p<0,05)$, *Valores expresados en media $\pm \mathrm{DE},{ }^{* *}$ prueba $\chi^{2}$. 
Tabla 2. Ingesta de edulcorantes (mg/día) y porcentaje de adecuación IDA según país y sexo

\begin{tabular}{|lllll|}
\hline & Perú & Chile & Guatemala & Panamá \\
Mujeres & $\mathrm{n}=140$ & $\mathrm{n}=317$ & $\mathrm{n}=217$ & $\mathrm{n}=216$ \\
Acesulfamo de potasio (mg) & $0,72 \pm 1,00$ & $0,64 \pm 0,56$ & $0,61 \pm 1,11^{\mathrm{a}}$ & $0,76 \pm 1,04^{\mathrm{a}}$ \\
Aspartamo (mg) & $2,13 \pm 3,37$ & $1,96 \pm 4,75$ & $1,66 \pm 2,93^{\mathrm{a}}$ & $2,27 \pm 3,41^{\mathrm{a}}$ \\
Sucralosa (mg) & $0,88 \pm 1,33$ & $1,38 \pm 2,24^{\mathrm{a}}$ & $0,74 \pm 1,56$ & $0,38 \pm 0,56^{\mathrm{a}}$ \\
\% Adecuación IDA Acesulfamo de potasio & 4,8 & 4,2 & 4,0 & 5,0 \\
\% Adecuación IDA Aspartamo & 4,2 & 3,9 & 3,3 & 4,5 \\
\% Adecuación IDA Sucralosa & 5,8 & 9,2 & 4,9 & 2,5 \\
Hombres & $\mathrm{n}=64$ & $\mathrm{n}=155$ & $\mathrm{n}=36$ & $\mathrm{n}=84$ \\
Acesulfamo de potasio (mg) & $0,82 \pm 1,10$ & $0,53 \pm 0,58^{\mathrm{a}}$ & $1,16 \pm 1,09^{\mathrm{a}}$ & $0,81 \pm 1,00$ \\
Aspartamo (mg) & $2,12 \pm 3,10$ & $1,45 \pm 2,36^{\mathrm{a}}$ & $3,04 \pm 3,22^{\mathrm{a}}$ & $2,03 \pm 3,13$ \\
Sucralosa (mg) & $0,73 \pm 1,35$ & $0,67 \pm 1,10$ & $0,68 \pm 0,58$ & $0,36 \pm 0,65$ \\
\% Adecuación IDA Acesulfamo de potasio & 5,4 & 3,1 & 7,7 & 5,4 \\
\% Adecuación IDA Aspartamo & 4,2 & 2,9 & 4,0 & 4,0 \\
\% Adecuación IDA Sucralosa & 4,8 & 4,4 & 2,4 & 2,4 \\
\hline
\end{tabular}

Prueba de Anova post hoc Bonferroni, letras iguales indican diferencias significativas $(p<0,05)$ *Valores expresados en media \pm DE.

to en el riesgo de sobrepeso/obesidad $\mathrm{OR}=10,0$ (IC $=1,2-78,1 ; p=0,02)$ (Tabla 3). Todos los modelos fueron ajustados por edad, pero esta variable no influyó en forma significativa en ningún modelo ( $\mathrm{p}>0,05)$.

\section{Discusión}

Nuestro principal resultado fue que en universitarias latinoamericanas el consumo de acesulfame de potasio y sucralosa disminuye el riesgo de sobrepeso/obesidad. En cambio, los otros edulcorantes evaluados, tanto en mujeres como en hombres, no se asocian con riesgo de sobrepeso/ obesidad, excepto en hombres guatemaltecos cuyo consumo sí incrementa el riesgo y son quienes presentan un mayor consumo de ENN.

Es sabido que el azúcar proporciona una gran cantidad de hidratos de carbono que se absorben rápidamente y que lleva a la ingesta excesiva de energía, aumento de peso y síndrome metabóli$\mathrm{Co}^{16-17}$. La mayoría de la gente elige los ENN para perder o mantener el peso.

Sin embargo, estudios no aleatorios, transversales y longitudinales han reportado una asociación entre los ENN y el incremento en el peso corporal aunque con resultados contradictorios, situación similar presentada en nuestro estudio con el consumo de acesulfamo de potasio en Guatemala $^{18}$. Estos resultados son difíciles de interpretar ya que no entregan causalidad. Los estudios transversales no pueden determinar si las personas que incrementan el peso comienzan a utilizar edulcorantes para frenar el incremento de peso o bajar de peso. En general los investigadores concluyen que no hay evidencia significativa de estudios de observación que asocien los ENN con el aumento de peso ${ }^{19}$.

Estudios experimentales a corto plazo han examinado la ingesta de alimentos después de precargas con ENN en bebidas y alimentos, aunque los resultados han sido contradictorios en relación al apetito ${ }^{20-22}$. Un estudio realizado en humanos por Bryant y cols. ${ }^{23}$ mostró que ENN como aspartamo y sacarina, entregados en dosis de consumo habitual sumado a glucosa, no ejercían un efecto adicional sobre la glicemia ni la percepción de hambre y saciedad. Sin embargo, aunque acesulfamo de potasio sí ejercía un efecto leve en el incremento de glucosa, los autores concluyeron que no hay evidencia consistente en cuanto al efecto del consumo de ENN sobre la glucosa, cuando se utilizan en cantidades de consumo habitual.

Revisiones concluyen que no existen datos que sugieran que el consumo de ENN presentes en alimentos o bebidas promueva la ingesta de alimentos y el incremento de peso $^{23}$, por otra parte, 
Edulcorantes y riesgo de obesidad en universitarios latinoamericanos - S. Durán Agúero et al

Tabla 3. Asociación entre el consumo de edulcorantes y el sobrepeso/obesidad*

\begin{tabular}{|c|c|c|c|c|}
\hline Variables & OR & \multicolumn{2}{|c|}{ IC al 95\% } & Valor $\mathbf{p}$ \\
\hline \multicolumn{5}{|c|}{ Edulcorantes (suma todo los países) } \\
\hline Total ENN & 0,2 & 0,029 & 3,060 & 0,29 \\
\hline Acesulfame de potasio & 0,6 & 0,454 & 1,022 & 0,06 \\
\hline Aspartame & 0,9 & 0,698 & 1,387 & 0,92 \\
\hline Sucralosa & 0,7 & 0,488 & 1,061 & 0,09 \\
\hline \multicolumn{5}{|l|}{ Mujeres } \\
\hline Acesulfame de potasio & 0,5 & 0,3 & 0,9 & 0,03 \\
\hline Aspartame & 0,2 & 0,4 & 1,2 & 0,78 \\
\hline Sucralosa & 0,4 & 0,2 & 0,8 & 0,01 \\
\hline \multicolumn{5}{|l|}{ Hombres } \\
\hline Acesulfame de potasio & 0,8 & 0,4 & 1,5 & 0,52 \\
\hline Aspartame & 1,1 & 0,6 & 2,0 & 0,56 \\
\hline Sucralosa & 0,9 & 0,5 & 1,6 & 0,81 \\
\hline \multicolumn{5}{|l|}{ Perú } \\
\hline Total ENN & 0,5 & 0,2 & 1,1 & 0,09 \\
\hline Acesulfamo potasio & 0,1 & 0,0 & 1,5 & 0,14 \\
\hline Aspartamo & 0,1 & 0,0 & 1,6 & 0,12 \\
\hline Sucralosa & 0,8 & 0,3 & 1,7 & 0,60 \\
\hline \multicolumn{5}{|l|}{ Chile } \\
\hline Total ENN & 0,7 & 0,4 & 1,2 & 0,34 \\
\hline Acesulfamo potasio & 1,2 & 0,6 & 2,6 & 0,51 \\
\hline \multicolumn{5}{|l|}{ Aspartamo } \\
\hline Sucralosa & 0,7 & 0,4 & 1,2 & 0,29 \\
\hline \multicolumn{5}{|l|}{ Guatemala } \\
\hline Total ENN & 2,8 & 0,3 & 22,8 & 0,30 \\
\hline Acesulfamo potasio & 10,0 & 1,2 & 78,1 & 0,02 \\
\hline Aspartamo & 1,4 & 0,5 & 3,4 & 0,42 \\
\hline Sucralosa & 2,8 & 0,8 & 9,9 & 0,09 \\
\hline \multicolumn{5}{|l|}{ Panamá } \\
\hline Total ENN & 1,2 & 0,7 & 2,2 & 0,34 \\
\hline Acesulfamo potasio & 1,9 & 0,2 & 13,9 & 0,51 \\
\hline Aspartamo & 1,9 & 0,1 & 31,6 & 0,63 \\
\hline Sucralosa & 1,2 & 0,7 & 2,1 & 0,39 \\
\hline
\end{tabular}

*Valores ajustados por edad.

Popkin y Mattes ${ }^{16,21}$ llegaron a la conclusión que la sustitución de ENN produce una compensación calórica incompleta, pero no hay evidencia a largo plazo de la eficacia del uso de ENN para controlar el peso. Anderson y cols. ${ }^{24}$ concluyó que no hay pruebas de que los ENN puedan considerarse como causantes del aumento del peso corporal en el adulto. Finalmente un metaanálisis realizado por Miller y cols. ${ }^{25}$ indica que el consumo de ENN en estudios aleatorizados y controlados muestra una modesta pero significativa reducción en el peso corporal (-0,8 kg; IC 95\% -1,17- -0,43), IMC
$(-0,24 ;$ IC $95 \%-0,41--0,07)$, masa grasa $(-1,1 \mathrm{~kg}$; IC $95 \%-1,77--0,44)$ y circunferencia de cintura $(-0,8 \mathrm{~cm}$, IC $95 \%-1,29--0,37)$. Por otro lado, los estudios de cohorte prospectivo la ingesta de ENN no se asoció con el peso y masa grasa, pero sí con un incremento ligero del IMC $(0,03$; IC 95\% 0,01-0,06), los autores concluyen que el consumo de ENN es una herramienta útil para la dieta para mejorar planes dietéticos hipocalóricos. Sin embargo, hay que considerar que la obesidad presenta una naturaleza multifactorial. Entre los diversos factores que condicionan la obesidad 
destacan factores genéticos, metabólicos, psicológicos, neuroendocrinos ${ }^{26}$, una ingesta calórica elevada y una disminución del gasto energético por actividad física, los cuales con frecuencia actúan de forma conjunta son los principales factores de riesgo para el desarrollo de obesidad ${ }^{27}$. Sumado a lo anterior, hay factores que pueden fomentar una alimentación excesiva como por ejemplo la restricción de sueño ${ }^{28,29}$.

Entre las limitaciones del presente estudio, está que no es una muestra aleatoria y que es un estudio transversal, por lo tanto no podemos hablar de causalidad, sino de asociación. Además el modelo no fue ajustado por actividad física, ingesta alimentaria, consumo de tabaco y cantidad de sueño, factores que podrían influir en el estado nutricional de los sujetos. Por otro lado, tampoco fue posible hacer un análisis de sensibilidad y especificidad de la encuesta y debe mencionarse también que con cualquier estudio hecho con métodos no objetivos, el sesgo de deseabilidad social es posible. Esto reduce nuestra habilidad de confiar en los niveles de consumo reportado.

Entre las fortalezas, podemos nombrar que es una muestra latinoamericana, es el primer estudio realizado en países en transición nutricional, que se utilizó una encuesta validada y con amplia variedad de ENN disponibles en el mercado de cada país, incluyendo ENN líquidos, en polvo y en tabletas de gran uso en infusiones (café) y otras preparaciones de alto consumo en los universitarios.

\section{Conclusión}

En mujeres, el consumo de sucralosa y acesulfame de potasio aparecen como un factor protector frente a obesidad. Evidentemente, hay otros factores como el ejercicio, sedentarismo y el consumo de alcohol, entre otros, que influyen el riesgo de obesidad en una población universitaria. Nuestros resultados, junto con el aumento de consumo de los edulcorantes en América Latina en los últimos años y las altas prevalencias altas de obesidad en la región, muestran una necesidad apremiante de estudios futuros sobre el rol de los edulcorantes en el riesgo sobrepeso y obesidad.

Agradecimientos: A todo el personal que trabajó en el estudio.

\section{Referencias}

1. Vio F, Albala C. Nutrition Policy in the Chilean Transition. Public Health Nutrition 2000; 3 (1): 49-55.

2. Pérez RC. Current mapping of obesity. Nutr Hosp 2013; 28: 21-31.

3. Filozof C, González C, Sereday M, Mazza C, Braguinsky J. Obesity prevalence and trends in Latin-American countries. Obes Rev 2001; 2: 99-106.

4. Rosell M, Hakansson NN, Wolk A. Association between dairy food consumption and weight change over $9 \mathrm{y}$ in 19,352 perimenopausal women. Am J Clin Nutr 2006; 84: 1481

5. Zemel MB. Mechanisms of dairy modulation of adiposity. J Nutr 2003; 133: S252-6.

6. Calorie Control Council. The benefits of reduced caloric foods and beverages in weight management. Atlanta: Calorie Control Council; 2010. Available at: http://www. caloriecontrol.org.

7. Durán S, Quijada M, Silva L, Almonacid N, Berlanga M, Rodríguez MP. Niveles de ingesta diaria de edulcorantes no nutritivos en escolares de la región de Valparaíso. Rev Chil Nutr 2011; 38: 444-9.

8. Zanini R, Araújo C, Martínez-Mesa J. Utilização de adoçantes dietéticos entre adultos em Pelotas, Rio Grande do Sul, Brasil: um estudo de base populacional. Cad Saúde Pública 2011; 27: 924-34.

9. Zygler A, Wasik A, Wasik AK, Namiesnik J. Determination of nine high-intensity sweeteners in various foods by high-performance liquid chromatography with mass spectrometric detection. Anal Bioanal Chem 2011; 400: 2159-72.

10. Bellisle F, Drewnowski A. Intense sweeteners, energy intake and the control of body weight. Eur J Clin Nutr 2007; 61: 691-700.

11. Mattes R, Popkin B. Nonnutritive sweetener consumption in humans: effects on appetite and food intake and their putative mechanisms. Am J Clin Nutr 2009; 89: 1-14.

12. Peters JC, Wyatt HR, Foster GD, Pan Z, Wojtanowski AC, Vander Veur SS, et al. The effects of water and nonnutritive sweetened beverages on weight loss during a 12-week weight loss treatment program. Obesity (Silver Spring). 2014; 22 (6): 1415-21.

13. Swithers SE. Artificial sweeteners produce the counterintuitive effect of inducing metabolic derangements. Trends Endocrinol Metab 2013; 24 (9): 431-41.

14. Arcella D, Le Donne C, Piccinelli R, Leclercq C. Dietary estimated intake of intense sweeteners by Italian teenagers. Present levels and projections derived from the INRAN-RM 2001 food survey. Food Chem Toxicol 2004; 42: 677-85. 
15. Reglamento Sanitario de los Alimentos. http://web.minsal.cl/sites/default/files/2013RSADECRETO_977_96_actualizado2013.pdf

16. Popkin BM, Nielsen SJ. La edulcoración de la dieta mundial. Res Obes 2003; 11: 1325-32.

17. Schulze MB, Manson JE, Ludwig DS, Colditz GA, Stampfer MJ, Willett WC. et al. Sugar-sweetened beverages, weight gain, and incidence of type 2 diabetes in young and middle-aged women. JAMA 2004; 292: 927-34.

18. Serra-Majem L, Ribas L, Ingles C, Fuentes M, Lloveras G, Salleras L. Cyclamate consumption in Catalonia, Spain (1992): relationship with the body mass index. Food Addit Contam 1996; 13: 695-703.

19. Butchko HH, Stargel WW, Comer CP, Mayhew DA, Benninger C, Blackburn GL, et al. Aspartame: review of safety. Regul Toxicol Pharmacol 2002; 35: S1-93.

20. Phelan S, Lang W, Jordan D, Wing RR. Use of artificial sweeteners and fat-modified foods in weight loss maintainers and always normal weight individuals. Int J Obes (Lond). 2009; 33: 1183-90.

21. Mattes RD, Popkin BM. Nonnutritive sweetener consumption in humans: effects on appetite and food intake and their putative mechanisms. Am J Clin Nutr 2009; 89: $1-14$.

22. Rolls BJ, Kim S, Fedoroff IC. Effects of drinks sweetened with sucrose or aspartame on hunger, thirst and food intake in men. Physiol Behav 1990; 48: 19-26.

23. Bryant CE, Wasse LK, Astbury N, Nandra G, McLaughlin JT. Non-nutritive sweeteners: no class effect on the glycaemic or appetite responses to ingested glucose. Eur J Clin Nutr 2014; 68 (5): 629-31.

24. Anderson GH, Foreyt J, Sigman-Grant M, Allison DB. The use of low-calorie sweeteners by adults: impact on weight management. J Nutr 2012; 142 (6): 1163S-9S.

25. Miller PE, Pérez V. Low-calorie sweeteners and body weight and composition: a meta-analysis of randomized controlled trials and prospective cohort studies. Am J Clin Nutr 2014; 100 (3): 765-77.

26. Carrero I, Rupérez E, Miguel R. de Tejero JA, PérezGallardo L. Ingesta de macronutrientes en adolescentes escolarizados en Soria capital. Nutr Hosp 2005; 20 (3): 204-9.

27. Uauy R. Atalah E, Kain J. The nutritional transition: new nutritional Influences on child growth. In: Martorell R, Haschke F, editors Nutrition and growth. Williams and Wilkins, Philadelphia, USA, 2001; pp 305-28.

28. Chamorro RA, Durán SA, Reyes SC, Ponce R, Algarín CR, Peirano PD. Sleep deprivation as a risk factor for obesity. Rev Med Chile 2011; 139 (7): 932-40.

29. Chapman CD, Benedict C, Brooks SJ, Schiöth HB. Lifestyle determinants of the drive to eat: a meta-analysis. Am J Clin Nutr 2012; 96 (3): 492-7. 